University of Wollongong

Research Online

Faculty of Engineering and Information

Faculty of Engineering and Information

Sciences - Papers: Part A

Sciences

$1-1-2014$

Over-voltage mitigation within distribution networks with a high renewable distributed generation penetration

Joel Kennedy

University of Wollongong, jtk847@uowmail.edu.au

Philip Ciufo

University of Wollongong, ciufo@uow.edu.au

Ashish Agalgaonkar

University of Wollongong, ashish@uow.edu.au

Follow this and additional works at: https://ro.uow.edu.au/eispapers

Part of the Engineering Commons, and the Science and Technology Studies Commons

Research Online is the open access institutional repository for the University of Wollongong. For further information contact the UOW Library: research-pubs@uow.edu.au 


\title{
Over-voltage mitigation within distribution networks with a high renewable distributed generation penetration
}

\begin{abstract}
The rapid growth of grid-connected distributed generation has increased the likelihood of over-voltage occurrences in distribution networks. In recent times, much research has taken place in order to develop a control strategy to mitigate the voltage rise problem. However, most of the published strategies require retuning when additional resources are connected, or have a strong dependence on network parameters, such as fault level. This paper proposes a novel over-voltage mitigation scheme that has many advantages not observed in literature. Firstly, the control scheme can integrate with an existing feeder in a plug-and-play fashion. No prior analysis is necessary to configure the control parameters; all required information is measured locally. Secondly, the control scheme is a simple extension upon constant power control which is common in most grid-connected inverter interfaces. Finally, the proposed over-voltage mitigation scheme enforces a fair and equitable power flow allocation. The scheme contains a predefined point of convergence for any voltage magnitude measured at the point of common coupling. Many control schemes operate in a perturb and observe manner which can inadvertently allow certain DG units to export a disproportionate amount of power with respect to other DG units. This paper also details a methodology for analysing the cost effectiveness of any given DG configuration utilising the proposed over-voltage mitigation scheme. The analysis is useful for determining whether a network infrastructure upgrade may be necessary as power curtailment becomes more prevalent within a distribution network. 2014 IEEE.
\end{abstract}

\section{Keywords}

renewable, distributed, generation, penetration, distribution, networks, mitigation, over, high, voltage, within

\section{Disciplines \\ Engineering | Science and Technology Studies}

\section{Publication Details}

J. Kennedy, P. Ciufo \& A. Agalgaonkar, "Over-voltage mitigation within distribution networks with a high renewable distributed generation penetration," in Energy Conference (ENERGYCON), 2014 IEEE International, 2014, pp. 1107-1114. 


\title{
Over-Voltage Mitigation within Distribution Networks with a High Renewable Distributed Generation Penetration
}

\author{
Joel Kennedy ${ }^{1}$, Phil Ciufo ${ }^{2}$, Ashish Agalgaonkar ${ }^{3}$ \\ School of Electrical, Computer \\ and Telecommunications Engineering, \\ University of Wollongong, \\ Wollongong, NSW, Australia \\ 1 jtk847@uowmail.edu.au \\ ${ }^{2}$ ciufo@uow.edu.au \\ 3 ashish@uow. edu. au
}

\begin{abstract}
The rapid growth of grid-connected distributed generation has increased the likelihood of over-voltage occurrences in distribution networks. In recent times, much research has taken place in order to develop a control strategy to mitigate the voltage rise problem. However, most of the published strategies require re-tuning when additional resources are connected, or have a strong dependence on network parameters, such as fault level. This paper proposes a novel over-voltage mitigation scheme that has many advantages not observed in literature. Firstly, the control scheme can integrate with an existing feeder in a plugand-play fashion. No prior analysis is necessary to configure the control parameters; all required information is measured locally. Secondly, the control scheme is a simple extension upon constant power control which is common in most grid-connected inverter interfaces. Finally, the proposed over-voltage mitigation scheme enforces a fair and equitable power flow allocation. The scheme contains a predefined point of convergence for any voltage magnitude measured at the point of common coupling. Many control schemes operate in a perturb and observe manner which can inadvertently allow certain DG units to export a disproportionate amount of power with respect to other DG units. This paper also details a methodology for analysing the cost effectiveness of any given DG configuration utilising the proposed over-voltage mitigation scheme. The analysis is useful for determining whether a network infrastructure upgrade may be necessary as power curtailment becomes more prevalent within a distribution network.
\end{abstract}

Index Terms-Over-voltage, distributed generation, inverters, distribution networks.

\section{INTRODUCTION}

The recent growth of inverter-interfaced grid-connected distributed generation (DG) in Australian distribution networks (DNs) has been a contentious issue within the power community of late. There are many advantages of grid-connected DG,

NOTICE: this is the authors' version of a work that was accepted for publication. Changes resulting from the publishing process, such as peer review, editing, corrections, structural formatting, and other quality control mechanisms may not be reflected in this document. Changes may have been made to this work since it was submitted for publication A definitive version was subsequently published in the Proceedings of the IEEE International Energy Conference, EnergyCon2014, May 2014, DOI:10.1109/ENERGYCON.2014.6850562 including promoting the use of renewable energy resources, improving fault ride-through, reducing losses in the DN and deferring infrastructure upgrades for the utility. However, there are also various potential complications involved with grid-connected DG. Most notably, power quality problems such as over-voltage (OV) may arise as well as protection maloperation [1], [2], [3].

Utilities have recorded instances of $\mathrm{OV}$ at the point of common coupling (PCC) of DG units and have subsequently imposed limits on the maximum size of a DG installation [4]. OV incidents generally occur under low local load conditions in networks where a significant short circuit impedance exists between the DG unit and the nearest upstream voltage regulated point. Grid-connected DG units do not explicitly regulate voltage. DG units most commonly regulate the output real power at unity power factor; the resultant frequency and voltage are line-commutated [5], [6].

Possible OV prevention methods in DNs with a significant DG presence have been investigated by various authors. These methods shall be reviewed in Section II. The difficulty of OV prevention arises through the conflict between the business case for DG installation and the technical requirements stipulated within the Australian and IEEE Standards. A customer wishes to output as much energy as possible to maximise their return. However, if a maximum power output results in an OV situation, the loss of equipment life is generally more expensive than the extra income earned throughout the low load condition period. To satisfy both the technical and economic demands of DG connection, many papers have discussed the use of reactive power absorption in order to mitigate the voltage rise while still allowing maximum apparent power output [1], [7]. This paper shall provide a novel control scheme for multiple DG units that ensures fair return on investment and maintains the voltage of the grid within the stipulated bounds expressed in [8].

Inverters have been adopted as the preferred DG grid interface within this paper for a variety of reasons. Firstly, inverters have become increasingly popular in Australian DNs; 
installations have dramatically increased due to government incentives and the cost of infrastructure and installation has consistently decreased over the last several years [9]. Secondly, the robust nature of inverter control is imperative for the advanced control schemes required to allow DNs to function as desired when a high DG penetration is present. Finally, the decoupling of the dynamic response of the energy resource from the grid is essential for seamless integration of the DN with the stochastic nature of renewable energy resources. Furthermore, more progressive notions such as the Microgrid concept require fast load following via droop control during intentional islanding operation which is best realised through use of an inverter interface [10], [11].

The remainder of this paper is organised as follows. Section II provides a review of the OV mitigation schemes proposed by various authors. Section III outlines the proposed OV mitigation scheme. A methodology for performing a cost analysis of a DN with DG implementing the new scheme in shown in Section IV. Section V contains the conclusions and recommendations.

\section{ReView of OV Mitigation Schemes IN DNS With High DG PENETRATION}

The simplest way to avoid OV in DNs with DG penetration is to place restrictions on DG size and location within the planning process. Australian utilities can impose a restriction on the size of a proposed DG unit upon application [12]. The current philosophy of DG control stipulates fixed power at unity power factor - a paradigm which suppresses the growth of DG penetration in Australian DNs. The worst case voltage profile can be determined by approximating the low load condition of a DN and running continuous load flow analyses for DG sizes until the voltage limit is breached. Such an exercise is time intensive and shown to be unnecessary through voltage sensitivity analysis as shown by Ayres et al. in [13].

Voltage sensitivity analysis manipulates the Jacobian matrix in order to predict the maximum DG size permissible before an OV is likely to arise. The Jacobian matrix is an array of the linearised rates of change tangential about a given operating point. For small deviations, linearisation can give a reasonably accurate representation of a network's expected performance. Ayres' work is extended upon in [14] where the loadability of a line is also considered to ensure the thermal limits of a distribution network are not exceeded under low load conditions. However, these papers do not explore the plausibility of an OV management scheme which could allow for a greater DG penetration. The authors in [1] use the sensitivity matrix to approximate the appropriate power factor for a DG unit in a worst case low load scenario. The sensitivity analysis is useful for determining a reasonably fair method for allocating the reactive power license of each DG unit with respect to that DG unit's position in a DN. Various reactive power control methods are outlined in [1] and [15] ranging from constant power factor, to real power-dependent power factor and voltage-dependent reactive power control. The principal weakness of these control strategies is the necessary reconfiguration whenever a network upgrade occurs or a new DG unit is installed.

Consider the scenario where each DG unit is to be coupled with an energy storage device such as batteries. In such a case, any surplus power produced by the distributed energy resources shall be diverted into the battery bank, thus eliminating the risk of producing an OV caused by exporting more power than is being absorbed locally. Under continuous low load conditions, it may be possible that the batteries may be filled to capacity. The power set points of the inverter interface must then be altered or the DG unit will trip due to the OV anti-islanding protection. Such a problem can be mitigated through the appropriate sizing and control of the battery bank. However, it is important to note that the most significant deterrence of the installation of battery banks remains the exorbitant cost and slow return rate. It is unlikely that most customers would invest in energy storage unless either the cost of storage is reduced or significant benefits were to arise such as the possibility of intentional islanding. Intentional islanding is forbidden by Australian Standard 4777.3 [8] and, as such, shall not be considered within this paper.

Another possible technique for OV mitigation is demandside load management. If load behaviour could be configured to coincide with times of maximum generation, the risk of $\mathrm{OV}$ could be greatly diminished. However, customer and load coordination can be particularly challenging as maximum DG availability can be unpredictable. Even in the case of solar irradiation being somewhat predictable due to the quotidian nature of our planet's rotation, the maximum solar irradiance tends to occur when people are at work. In a commercial site, this coincidence is fortunate, but for residential premises, there exists a disparity between load and generation availability. It is generally accepted that variable tariffs and consumer awareness can be used to curb power usage during peak times [16]. However, under low load conditions where OV instances are most likely, there may be little extraneous load that customers are willing to incur. The notable exception is the off-peak power usage of hot water systems; hot water water systems are capable of storing heat for large periods of time. While demand-side management can be implemented to ease the disparity between generation availability and demand during times of peak load, off-peak demand-side load management schemes will likely coalesce into an energy storage problem, yielding a situation similar to that explored previously with power diversion into battery banks. For similar reasons, demand-side management schemes shall not be considered within this paper.

Assuming that most customers do not invest in energy storage, the next most viable option for OV mitigation whilst maximising return is reactive power absorption. Currently, Australian DG unit proprietors are paid for real power injection only. There is a strong case that eventually reactive 
power may become a marketable commodity within DNs [17]; however, the notion of reactive power remuneration is unlikely to be prevalent in Australian DNs in the near future and hence the notion shall not be included in this paper. Without reactive power remuneration, it makes financial sense for a DG unit to absorb reactive power during OV to maintain the voltage within acceptable levels, rather than solely reducing the real power export. A small amount of real power output capacity is lost when reactive power is injected or absorbed by an inverter. The capability curve is derivative of the rated current restriction assuming the inverter is operating at rated voltage; a current restriction is implemented to prevent damage to the power electronic switches of the inverter interface. The utility imposes strict boundaries on the power factor produced by any DG unit; for example, 0.85 leading or lagging according to the standard IEEE 929 [18]. An OV mitigation scheme's configuration must be commensurate with these boundaries.

If the limitations of reactive power absorption are met, then the real power set point of the inverter interface must be limited as proposed by Calderaro et al. [7]. Calderaro et al. builds on the sensitivity matrix work presented by Ayres et al. in [13] to provide an OV mitigation algorithm. The algorithm accounts for both the voltage and current limitations of the DG unit. There are two main drawbacks to Calderaro et al.'s algorithm. Firstly, a knowledge of the sensitivity of the network is required and the control scheme needs to be reconfigured whenever a significant alteration occurs in the DN. Hence, the Calderaro system cannot be considered as 'plug-and-play'. Secondly, the perturbation and observation nature of the control scheme can make convergence to a preferred set point for each DG unit difficult and potentially produce disproportionate power flow allocations between DG units. In comparison, the OV mitigation protection scheme proposed within this paper requires only local readings and converges to a predefined point to ensure fair power flow within a DN.

\section{Novel OV Mitigation Algorithm}

The proposed OV mitigation algorithm is designed to ensure a fair power flow allocation using only locally sourced data. The algorithm incorporates the capabilities of the inverter interface, the available power from the distributed energy source as well as the stipulated requirements of the DN.

Firstly, a voltage limit shall be imposed as in (1).

$$
\left|\overrightarrow{V_{\mathrm{DG}}}\right| \leq V_{\text {limit }}
$$

Where $\left|\vec{V}_{\mathrm{DG}}\right|$ is the voltage magnitude at a DG unit's PCC and $V_{\text {limit }}$ is the voltage magnitude limit imposed by the OV mitigation algorithm. The voltage limit should be chosen to be below the OV threshold of the anti-islanding protection system to avoid nuisance tripping.
A power factor limit is also imposed in order to abide by standards such as the IEEE Standard 929 [18].

$$
\text { p.f. } \text { limit }_{\text {limit }}=\cos \left(\theta_{\text {limit }}\right) \leq \frac{P}{|\vec{S}|}
$$

Finally, the power capability curve of the inverter interface is represented as a semicircle. As mentioned briefly in the introduction, the capability curve is implemented by the control scheme to prevent damage to the power electronic switches. The domain of complex power operation is expressed in (3).

$$
\vec{S} \epsilon\left\{\vec{x}=\mathbb{C}:|\vec{x}| \leq\left|S_{\text {rated }}\right|, 0 \leq \arg (\vec{x}) \leq \theta_{\text {limit }}\right\}
$$

It is assumed within this paper that reactive power injection is forbidden. Ergo, the imaginary component of the complex power $\vec{S}$ will always be non-negative. The inclusion of reactive power injection shall be explored in future work.

Beyond the constraints defined in (1), (2) and (3), the control scheme must provide a fair and equitable solution to avoid OV and maximise real power output. Firstly, it must be understood that it is preferable to absorb reactive power before reducing real power below the value defined on the outermost limit of the power capability domain. It is only when the power factor limit is reached that the apparent power $|\vec{S}|$ should be deliberately curtailed. The power factor limit is maintained during apparent power curtailment, again with the purpose of maximising real power output.

Given that the available apparent power $S_{\text {available }}$ is known, the domain of steady-state operation can be reduced to (4).

$$
\vec{S} \epsilon\left\{\vec{x}=\mathbb{C}:|\vec{x}|=S_{\text {available }}, 0 \leq \arg (\vec{x})<\theta_{\text {limit }}\right\}
$$

Until real power curtailment is necessary to reduce the voltage when the domain changes to (5).

$$
\vec{S} \epsilon\left\{\vec{x}=\mathbb{C}: 0 \leq|\vec{x}| \leq S_{\text {available }}, \arg (\vec{x})=\theta_{\text {limit }}\right\}
$$

The available apparent power $S_{\text {available }}$ is capped at the rated apparent power of the system $S_{\text {rated }}$. Without some form of energy storage coupled to the DC side of the inverter, the available power may drop below the rated power of the inverter. In such a case, the value of available power can be sourced from the maximum power-point tracker (MPPT).

If a single DG unit is present within a grid, the control scheme could utilise a constant voltage magnitude limit. Reactive power absorption would begin once the voltage magnitude threshold was reached. The controller would operate in a perturb and observe fashion, where the maximum real power in achieved using the domains of (4) and (5) where the voltage limit is not exceeded satisfying (1). If the power factor limit was met and the voltage still exceeded the voltage magnitude threshold, then apparent power curtailment would begin until the voltage condition was satisfied. However, when multiple DG units are connected to a DN, a constant voltage threshold can incite power export contests. Without some form of communication medium, there is no way to prevent a set of DG units from inadvertently and needlessly forcing 


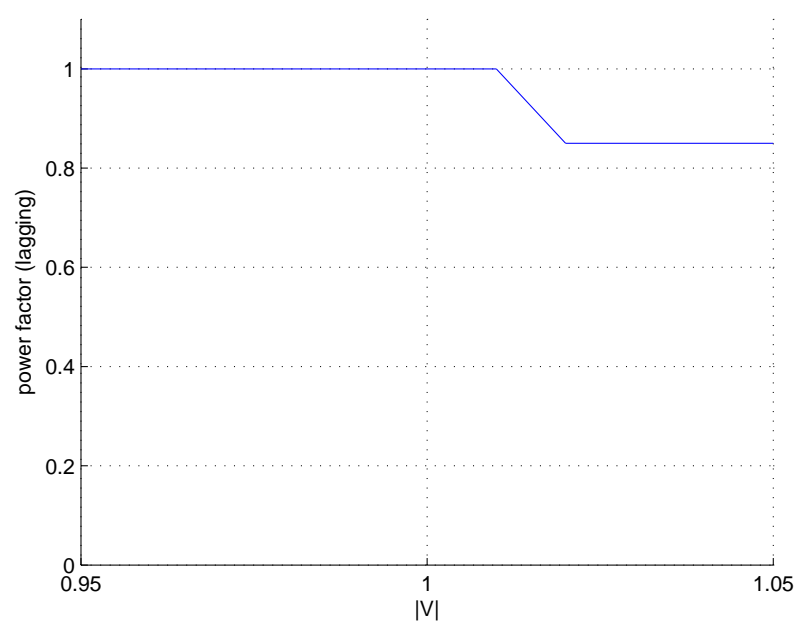

Fig. 1. Proposed OV Mitigation Scheme: Power Factor and Voltage Magnitude Relationship

other DG units into apparent power curtailment submission. There would also be an inherent stability problem with no clear point of convergence throughout this perturb and observe control scheme.

The proposed OV mitigation scheme uses piecewise equations to define the real and reactive power set points based on the voltage magnitude $|\vec{V}|$ at the PCC and the available apparent power $S_{\text {available }}$ sourced from the MPPT. The piecewise equations are defined in equations (6) and (7). The piecewise equations are segregated by three voltage magnitude threshold tiers $V_{1}, V_{2}$ and $V_{3}$. Example plots are shown in figures 1 and 2 using the data in table I.

TABLE I

EXAMPLE VALUES

\begin{tabular}{|c|c|}
\hline Parameter & Set Point \\
\hline$V_{1}$ & 1.01 p.u. \\
\hline$V_{2}$ & 1.02 p.u. \\
\hline$V_{3}$ & 1.03 p.u. \\
\hline p.f.limit & 0.85 \\
\hline$|\vec{S}|$ & 1 p.u. \\
\hline
\end{tabular}

$$
\begin{gathered}
\text { p.f. }=\left\{\begin{aligned}
1 & \text { if }|\vec{V}| \leq V_{1}, \\
\frac{1-\text { p.f. }{ }_{\text {limit }}}{V_{1}-V_{2}}\left(|\vec{V}|-V_{1}\right)+1 & \text { if } V_{1} \leq|\vec{V}| \leq V_{2}, \\
\text { p.f.limit } & \text { otherwise. }
\end{aligned}\right. \\
|\vec{S}|=\left\{\begin{aligned}
S_{\text {available }} & \text { if }|\vec{V}| \leq V_{2}, \\
\frac{S_{\text {available }}\left(|\vec{V}|-V_{3}\right)}{V_{2}-V_{3}} & \text { if } V_{2} \leq|\vec{V}| \leq V_{3}, \\
0 & \text { otherwise. }
\end{aligned}\right.
\end{gathered}
$$

The voltage thresholds $V_{1}, V_{2}$ and $V_{3}$ are predefined and common to all generators. The relationship $V_{\text {nominal }}<V_{1}<$ $V_{2}<V_{3}<=V_{\text {limit }}$ must be satisfied, but there is a considerable

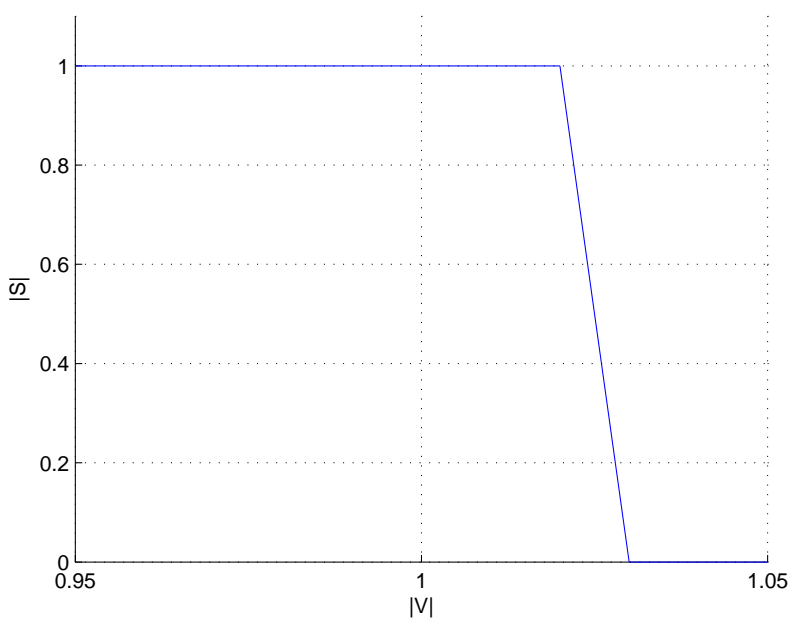

Fig. 2. Proposed OV Mitigation Scheme: Apparent Power and Voltage Magnitude Relationship

degree of freedom otherwise. The real and reactive set points can be determined from (8) and (9).

$$
P=|\vec{S}| \text { p.f. }
$$

$$
Q=|\vec{S}| \sqrt{1-\text { p.f. }^{2}}
$$

These power set points are then exported to a typical constant power inverter control system to determine the gating signals for the power electronic switches.

There are many advantages of the proposed OV mitigation scheme to those reviewed in Section II. Firstly, there is no need for a sensitivity analysis to be run to choose the set points for any stage of the algorithm. It must be stressed that the analysis provided in Section IV is only a tool for investigating the business case for DG installation and network infrastructure upgrades. The algorithm is not required for the normal operation of the proposed OV mitigation scheme.

Secondly, each DG unit can be installed and removed in a plug-and-play manner. No recalibration of any other control system is necessary. However, the expected return rate of a DG unit may change. Section IV defines a method to approximate the incurred gain or loss due to any alteration within a DN. The proposed OV mitigation scheme harnesses the stability and simplicity of constant PQ control and merely selects the P and $\mathrm{Q}$ set points based on the recorded voltage at the corresponding DG unit's PCC and the available apparent power from the distributed energy resource.

Finally, a clear point of convergence is defined by the piecewise equations, (6) and (7). The voltage threshold tiers must be common among all DG units within a feeder in order to ensure fair power distribution. While it may not be a completely egalitarian approach to power management, it is reasonable to allow upstream DG units with a low short circuit 
impedance to export more power than downstream DG units with a higher short circuit impedance. Voltage sensitivities show that upstream DG units are much less likely to produce an OV incident than downstream DG units. The piecewise equations (6) and (7) are intentionally designed to naturally allow better located DG units to export a greater real power than DG units considered more problematic.

\section{Steady-State Load Flow Modelling INCORPORATING THE OV MITIGATION ALGORITHM}

In order to perform an accurate cost analysis of DG unit installation, it is imperative to have an energy output forecasting tool. If a DG unit is susceptible to OV instances, an OV mitigation algorithm represents a loss of income. In order to determine this loss, load profiling and energy availability data can be integrated into a steady-state modelling tool to approximate the expected energy export curtailment throughout the life of the DG unit. This section shall provide that steady-state modelling tool.

The steady-state modelling tool begins with Newtonian linearisation in order to express the approximate voltage sensitivity of multiple DG units in a DN. The sensitivity matrix can be expressed as in (10). The Jacobian elements are the linearised rates of change tangential to a known operating point. The equations for the Jacobian elements are well established in the Newton-Raphson load flow algorithm. However, unlike the traditional Jacobian matrix, the non-diagonal elements of each quartile matrix (that relate separate elements' characteristics) are set to zero. The overall result is a two-by-two matrix relating $\Delta P$ and $\Delta Q$ which are the deviations in real and reactive power of a single $\mathrm{DG}$ unit respectively to $\Delta V$ and $\Delta \theta$ which are the deviations in voltage magnitude and voltage angular displacement of the same DG unit.

$$
\left[\begin{array}{l}
\Delta P \\
\Delta Q
\end{array}\right]=\left[\begin{array}{ll}
J_{P \theta} & J_{P V} \\
J_{Q \theta} & J_{Q V}
\end{array}\right]\left[\begin{array}{c}
\Delta \theta \\
\Delta V
\end{array}\right]
$$

The angular displacement of the voltage $\theta$ of a DG unit is not restricted by any standard or preferred mode of operation. Hence, simultaneous equations can be used to exclude this extraneous variable giving (11).

$$
\Delta P=J_{P \theta} J_{Q \theta}^{-1}\left(\Delta Q-J_{Q V} \Delta V\right)+J_{P V} \Delta V
$$

Equation (11) approximates the relationship between deviations in real and reactive power and deviations in voltage magnitude. This relationship is the cornerstone for determining the expected steady-state load flow solution incorporating the proposed OV mitigation algorithm.

The steady-state tool is essentially two Newtonian iterative solvers, one nested within another. The inner solver is simply the Newton-Raphson load flow program. The outer tool aims to assign the complex power output of each DG in a fair manner whilst satisfying the requirements stipulated in (1), (2) and (3) in accordance with the proposed OV mitigation scheme. The outer tool operates within four different states which are segregated by the three voltage threshold tiers $V_{1}$,
$V_{2}$ and $V_{3}$. The first state is solvable if a DG unit's voltage magnitude is less than $V_{1}$ under condition (12).

Firstly, the Newton-Raphson load flow shall be run with each DG unit exporting the maximum available power.

$$
\left|\overrightarrow{S_{\mathrm{DG}}}\right|=S_{\text {available }}
$$

If the voltage at the DG unit's PCC exceeds the first voltage threshold tier $V_{1}$ under operating condition (12), then the first state will be rejected and the second state shall be adopted where reactive power absorption shall be used to reduce the voltage magnitude at the PCC. The second state is solvable if $V_{1} \leq|V| \leq V_{2}$ is satisfied within the domain defined in (4): the maximum apparent power output shall remain constant and the power factor is varied between p.f. limit $_{\text {lagging and } 1 .}$

Given:

$$
|\vec{S}|^{2}=P^{2}+Q^{2}
$$

Linearisation about a known operating point $\left(P_{0}, Q_{0}\right)$ yields:

$$
0=P_{0} \Delta P+Q_{0} \Delta Q
$$

The 0 on the left hand side is a result of the apparent power being constant within the domain (4). The approximate desired voltage deviation $|\Delta V|$ can be constructed from the piecewise equation (6). Let:

$$
\Delta V=2\left(V_{\text {desired }}-V_{0}\right)
$$

$V_{0}$ is the magnitude of the voltage at the DG unit's PCC at a known operating point. The factor of two is a result of the equation (11) where an equal voltage deviation is assumed to result from the real and reactive power deviations. The voltage domain at this stage of the algorithm is $V_{1} \leq|\vec{V}| \leq V_{2}$. Hence, from (6):

$$
V_{\text {desired }}=\left(P_{0} / S_{\text {available }}+m_{1} V_{1}\right) / m_{1}
$$

Where:

$$
m_{1}=\frac{1-\text { p.f.limit }}{V_{1}-V_{2}}
$$

Substituting (14) into (15):

$$
\Delta V=2\left(\frac{P_{0} / S_{\text {available }}-1+m_{1} V_{1}}{m_{1}}-V_{0}\right)
$$

Combining (11), (13) and (16), the complete linearised system can be determined as shown in (17). If the reactive power $Q$ at the known operating point is zero, the system shall be reduced to (18).

If the power factor limit has been reached and the voltage threshold $V_{2}$ has been exceeded, the second state is rejected and the third state is introduced. The third state is solvable if a solution exists for $V_{2} \leq|\vec{V}| \leq V_{3}$ within the domain expressed in (5). An appropriately sized DG will rarely use this domain as real power curtailment can be severe and represent a significant loss of income. A heavily restricted power export can also create undesirable harmonic distortion. The required filter inductance to ensure harmonic distortion remains within acceptable levels is inversely proportional to 


$$
\begin{aligned}
& {\left[\begin{array}{ccc}
P_{0} & Q_{0} & 0 \\
1 & -\frac{J_{P \theta}}{J_{Q \theta}} & \frac{J_{P \theta} J_{Q V}}{J_{Q \theta}}-J_{P V} \\
1 & 0 & 0
\end{array}\right]\left[\begin{array}{c}
\Delta P \\
\Delta Q \\
\Delta V
\end{array}\right]=\left[\begin{array}{c}
0 \\
0 \\
2\left(\frac{P_{0} / S_{\text {avalable }}-1+m_{1} V_{1}}{m_{1}}-V_{0}\right)
\end{array}\right]} \\
& {\left[\begin{array}{cc}
1 & \frac{J_{P \theta} J_{Q V}}{J_{Q \theta}}-J_{P V} \\
1 & 0
\end{array}\right]\left[\begin{array}{l}
\Delta P \\
\Delta V
\end{array}\right]=\left[\begin{array}{c}
0 \\
2\left(\frac{P_{0} / S_{\text {avalable }}-1+m_{1} V_{1}}{m_{1}}-V_{0}\right)
\end{array}\right]} \\
& {\left[\begin{array}{ccc}
k & -1 & 0 \\
1 & -\frac{J_{P \theta}}{J_{Q \theta}} & \frac{J_{P \theta} J_{Q V}}{J_{Q \theta}}-J_{P V} \\
1 & 0 & 0
\end{array}\right]\left[\begin{array}{c}
\Delta P \\
\Delta Q \\
\Delta V
\end{array}\right]=\left[\begin{array}{c}
0 \\
0 \\
2\left(\frac{P_{0} / \text { p.f. }_{\text {limit }}+m_{2} V_{3}}{m_{2}}-V_{0}\right)
\end{array}\right]}
\end{aligned}
$$

the output power of an inverter [19]. It may be prudent to isolate the inverter when significant power curtailment occurs to ensure that significant harmonic distortion does not occur.

Beginning with:

$$
\tan \left(\cos ^{-1}\left(\text { p.f. } \text { limit }_{\text {lit }}\right)\right)=\frac{Q}{P}
$$

Linearisation yields:

$$
\Delta P\left(\tan \left(\cos ^{-1}\left(\text { p.f. } \text { limit }_{\text {. }}\right)\right)+\Delta Q(-1)=0\right.
$$

The power factor is constant within the domain (5). Equation (17) does not require the knowledge of initial operating points. An initial operating point of $\vec{S}=0$ will not produce a singularity upon solving, hence a second matrix is not required as in the second state.

The voltage domain at this stage of the algorithm is $V_{2} \leq$ $|V| \leq V_{3}$. Hence, from (7):

$$
V_{\text {desired }}=\left(P_{0} / \text { p.f. } \text {.limit }_{\text {. }}+m_{2} V_{3}\right) / m_{2}
$$

where:

$$
m_{2}=\frac{S_{\text {available }}}{V_{2}-V_{3}}
$$

Substituting (20) into (14):

$$
\Delta V=2\left(\frac{P_{0} / \text { p.f. } \text { limit }_{\text {in }}+m_{2} V_{3}}{m_{2}}-V_{0}\right)
$$

Combining (11), (18) and (21), another complete linearised system can be determined as shown in (22), where $k=$ $\tan \left(\cos ^{-1}(\right.$ p.f. limit $\left.)\right)$. The fourth and final state is an export of no power at which point the inverter will likely isolate itself from the grid via anti-islanding protection. This state should never be utilised during healthy operation of the grid and should be avoided through appropriate network planning.

The outer tool shifts between states as necessary based on the resultant magnitude of the voltage at the DG's PCC until a satisfactory operating point is found. Once the algorithm for determining the steady-state value of a DG unit has been completed, a simple cost analysis can be undertaken using (22).

$$
L=R \int_{-\infty}^{\infty} P(t) d t
$$

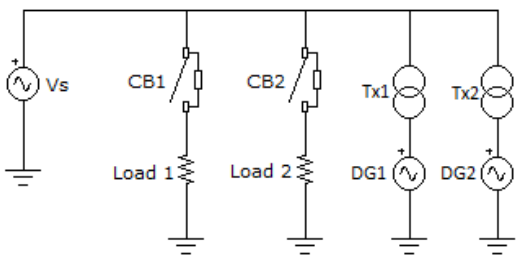

Fig. 3. Example Network

Where $L$ and $R$ are the total loss $(\$)$ and pay rate $(\$ / \mathrm{kWh})$ respectively and $P(t)$ in the power loss across time $(\mathrm{kW})$. If this cost is greater than the cost of an infrastructure upgrade (minus the losses under the upgrade condition) then an infrastructure upgrade is economically viable. It is important to note that the anticipated losses incurred are dependent on the growth of the feeder. For example, an increased DG penetration could represent a greater net loss for all DG proprietors. On the other hand, if load growth exceeds DG growth, losses are likely to be reduced. The net loss calculation is useful for determining when a network infrastructure upgrade is cost effective. There are also various other benefits in upgrade network infrastructure such as increased fault reach and reduced line over-loading which should be taken into account during the network planning process.

\section{Simulation}

In order to demonstrate the plausibility of the OV mitigation control scheme in practical application, a very simple network is constructed as shown in Figure 3. The simulation software has been composed by the author within the Matlab environment. The simulation is run twice, once with both DG units implementing constant power control and again using the OV mitigation scheme presented within this paper.

The line and load data is presented in the appendix in Table II. The infinite bus is operating at a voltage of 1.005 per unit in an $11 \mathrm{kV}$ network. The transformer and DG data is shown in the appendix in Table III and Table IV. Each DG has an LCL filter. The grid side inductor is simply a 


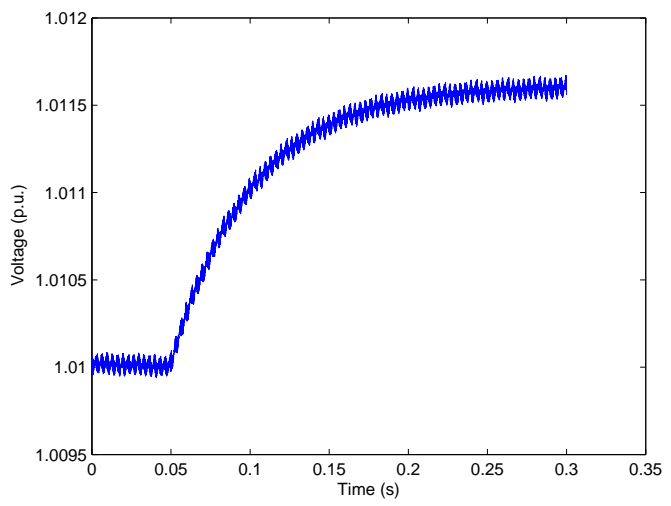

Fig. 4. $D G_{2}$ filtered voltage recording without OV mitigation

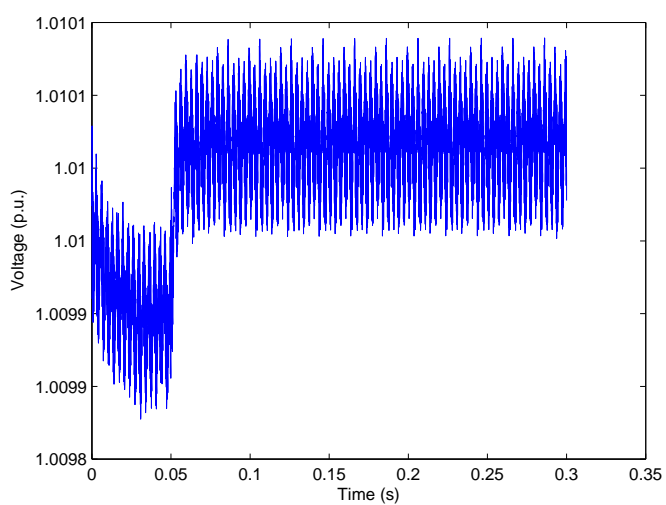

Fig. 5. $D G_{2}$ filtered voltage recording with OV mitigation

distribution transformer.

Load 1 is permanently connected to the network. Load 2 is isolated at 0.05 seconds. Without load 2, both DG units have to export power into the infinite bus in order to output a constant power. The resultant voltage magnitude at $D G_{2}$ as shown in Figure 4. A clear increase in steady state peak voltage can be observed after the isolation of Load 2.

The same situation is simulated implementing the OV mitigation scheme proposed within this paper. The new resultant voltage magnitude at $D G_{2}$ as shown in Figure 5.

A close analysis of Figure 5 shows the average voltage after the isolation of Load 2 to be approximately 1.0101 p.u. Using the piecewise equations (6) and (7), the power factor and apparent power set points can be calculated. The expected real and reactive power output can be determined to be $49.925 \mathrm{KW}$ and $2.737 \mathrm{kVAr}$ respectively. The real power output is shown in Figure 6. The average power was recorded to be approximately $49.95 \mathrm{~kW}$. The resultant reactive power is presented in Figure 7. The average reactive power is shown to be approximately $2.75 \mathrm{kVAr}$ as expected. The noise within both of the power waveforms is due to the measuring point of the voltage being located on the DG side of each distribution

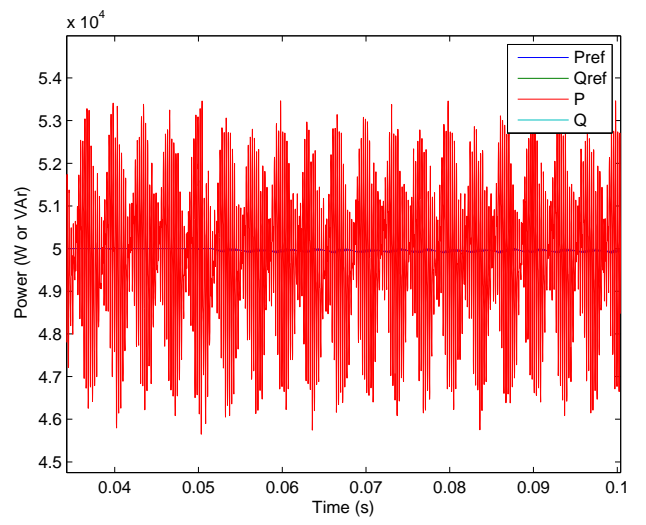

Fig. 6. $D G_{2}$ real power recording with $\mathrm{OV}$ mitigation

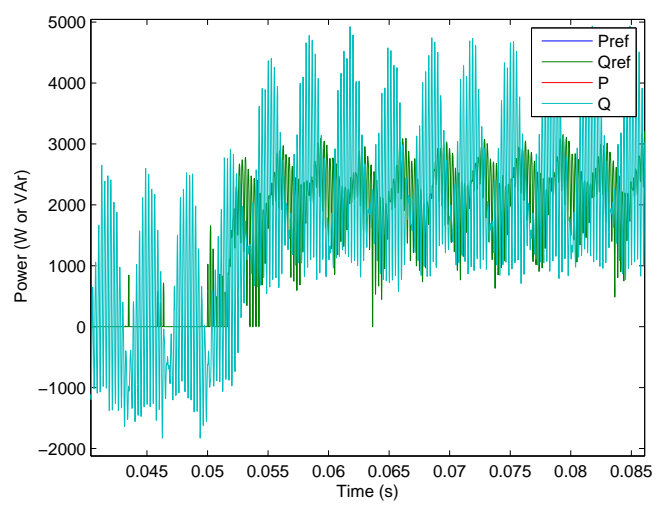

Fig. 7. $D G_{2}$ reactive power recording with $\mathrm{OV}$ mitigation

transformer.

These results are not intended to be a comprehensive review of the proposed OV mitigation scheme; rather an exploration into the plausibility of using piecewise equations to govern the real and reactive set points of inverter interfaced DG such that the most cost-effective result is obtained within any predefined voltage restrictions. The results show that the voltage was in fact curtailed under the low load condition using reactive power absorption when the first voltage threshold was reached. The set points chosen in this study are arbitrary and can be altered to better suit the economic and power quality requirements of the network.

\section{CONCLUSION}

A simple and practical OV mitigation scheme has been proposed within this paper. There are many advantages of the proposed scheme over a variety of other control schemes, including: plug-and-play compatibility, no need for communications, simple integration with existing constant power control schemes for inverters and a fair and equitable system for power flow allocation. Possible complications may arise where DG penetration reaches a significantly high percentage of the energy supply within a feeder. For example, if an island was to be formed when DG penetration levels are over $100 \%$ 
of the island, the OV mitigation scheme would prevent an OV situation from occurring that would normally be detected by anti-islanding protection. However, this problem is easily remedied if a significant reactive power requirement from the load cannot be met due to the loss of mains [20]. The DG control scheme within this paper does not allow voltage or reactive power support through reactive power injection of DG units. Hence, it is very likely that the anti-islanding protection would operation on under/over frequency or rate of change of frequency (ROCOF) protection. Failing those modes of protection, active anti-islanding methods such as vector shift will likely remove any non-detection zones [21], [22], [23].

Future work shall investigate the plausibility of incorporating line and transformer loading limits. Line and transformer loading becomes more problematic when reactive power is injected by DG units [14]. However, reactive power support is a useful tool for preventing voltages sags and is another topic for future consideration. The effects of apparent power curtailment on harmonic distortion also requires further research. It is envisaged that a more robust filter design may be necessary for significant power curtailment. Alternatively, an early trip may be warranted if the harmonic distortion becomes too great. Further, the implications of OV mitigation schemes on overcurrent protection operation are generally not well understood. The presence of DG penetration has been restricted in the last few years in Australia due to the presence of OV. Once $\mathrm{OV}$ issues no longer pose a threat to the healthy operation of Australian DNs, protection maloperation will likely become the next limiting factor in DG proliferation.

\section{APPENDIX}

TABLE II

EXAMPLE NETWORK VALUES

\begin{tabular}{|c|c|}
\hline Lines & Impedance $(\Omega)$ \\
\hline Line 1 & $0.5+j 0.125$ \\
\hline Line 2 & $0.5+j 0.125$ \\
\hline Line 3 & $1+j 0.125$ \\
\hline Line 4 & $10+j 0.0314$ \\
\hline Loads & Complex Power S $(k V A)$ \\
\hline Load 1 & 50 \\
\hline Load 2 & 200 \\
\hline
\end{tabular}

TABLE III

TRANSFORMER DATA

\begin{tabular}{|c|c|}
\hline Voltages $\left(V_{l-l r m s}\right)$ & $11000 / 400$ \\
\hline Primary Winding Impedance $(\Omega)$ & $0.01+j 0.31416$ \\
\hline Secondary Winding Impedance $(\Omega)$ & $0.01+j 0.31416$ \\
\hline Magnetising Branch Impedance $(\Omega)$ & $100000+j 28274334$ \\
\hline Connection & Star/Delta \\
\hline
\end{tabular}

\section{REFERENCES}

[1] Demirok, E. et al., "Local Reactive Power Control Methods for Overvoltage Prevention of Distributed Solar Inverters in Low-Voltage Grids", IEEE Journal of Photovoltaics, VOL. 1, NO. 2, 2011, pp. 174-182

[2] Endeavour Energy Power Quality and Reliability Centre, "Small Scale Domestic Rooftop Solar Photovoltaic Systems”, Technical Note 10, 2011
TABLE IV

DG DATA

\begin{tabular}{|c|c|c|}
\hline DG number & $D G_{1}$ & $D G_{2}$ \\
\hline Power Rating (kVA) & 100 & 50 \\
\hline AC Voltage $\left(V_{l-l r m s}\right)$ & 400 & 400 \\
\hline DC Voltage $V$ & 800 & 800 \\
\hline Switching frequency (Hz) & 4000 & 4000 \\
\hline DG-side Filter Inductance (mH) & 3.5 & 6.9 \\
\hline Filter Capacitance $(\mu$ F) & 6.217 & 3.1085 \\
\hline OV mitigiation V1 (p.u.) & 1.01 & 1.01 \\
\hline OV mitigiation V2 (p.u.) & 1.02 & 1.02 \\
\hline OV mitigiation V3 (p.u.) & 1.03 & 1.03 \\
\hline
\end{tabular}

[3] Conti, S., "Analysis of distribution network protection issues in presence of dispersed generation", Electric Power Systems Research, VOL. 79, NO. 1, 2009, pp. 49-56

[4] Hepworth, A., "Rooftop solar panels overloading electricity grid", The Australian, 13th of October, 2011

[5] Baran, M.E. and El-Markaby, I., "Fault analysis on distribution feeders with distributed generators", IEEE Transactions on Power Systems, VOL. 20, NO. 4, 2005, pp. 1757-1764

[6] Shang, W. and Redfern, M.A., "Control schemes for distributed generators connected to distribution networks", 2009 Proceedings of the 44th International Universities Power Engineering Conference (UPEC), 2009, pp. 1-5

[7] Calderaro, V. et al., "Optimal Decentralized Voltage Control for Distribution Systems With Inverter-Based Distributed Generators", IEEE Transactions on Power Systems, 2013

[8] Standards Australia, "Australian Standard AS 4777.3-2005, Standard for Grid Connection of Energy Inverter Systems via Inverter, Part 3: Grid Protection Requirements", 2005

[9] Watt, M. and Passey, R., "PV in Australia 2012: Executive Report", Australian PV Association, 2013

[10] Zeineldin, H.H. and El-Saadany, E.F. and Salama, M.M.A., "Distributed Generation Micro-Grid Operation: Control and Protection", Power Systems Conference: Advanced Metering, Protection, Control, Communication, and Distributed Resources, 2006. PS '06, 2006, pp.105-111

[11] Lasseter, R.H. et al., "The CERTS Microgrid Concept", White Paper for Transmission Reliability Program, Office of Power Technologies, U.S. Department of Energy, 2002

[12] AusGrid, Requirements for Connection of Embedded Generators, ES 11,2011

[13] Ayres, H.M. et al., "Method for determining the maximum allowable penetration level of distributed generation without steady-state voltage violations", Generation, Transmission Distribution, IET, VOL. 4, NO. 4, 2010, pp. 495-508

[14] Karimi-Zare, P. and Seifi, H., "Maximum allowable penetration level determination of a DG in a distribution network", 2012 IEEE Internationa Energy Conference and Exhibition (ENERGYCON), 2012, pp. 355-360

[15] Braun, M. et al., "Optimal reactive power supply in distribution networks: Technological and economic assessment for PV systems", 24th Eur. Photovoltaic Solar Energy Conf., Hamburg, Germany, 2009.

[16] Davito, B., "The Smart Grid and the Promise of Demand-Side Management", McKinsey and Company, 2010

[17] Abraham, E. et al., "Reactive Power Interconnection Requirements for PV and Wind Plants: Recommendations to NERC", SANDIA REPORT SAND2012-1098, 2012

[18] The Institute of Electrical and Electronics Engineers, Inc.,"IEEE Standards 929-2000, Recommended Practice for Utility Interface of Photovoltaic Systems"

[19] Biying, R. et al., "Analysis and design of an LCL filter for the threelevel grid-connected inverter", 2012 7th International Power Electronics and Motion Control Conference (IPEMC), 2012, Vol. 3, pp. 2023-2027

[20] Kennedy, J. and Ciufo, P. and Agalgaonkar, A., "Intelligent load management in Microgrids", 2012 IEEE Power and Energy Society General Meeting, 2012, p.p 1-8

[21] Ye Z. et al., "Study and Development of Anti-Islanding Control for GridConnected Inverters", National Renewable Energy Laboratory, 2004

[22] Ye Z. et al., "Grid-Connected Inverter Anti-Islanding Test Results for General Electric Inverter-Based Interconnection Technology", National Renewable Energy Laboratory, 2005 
[23] Bower, W. and Ropp, M.,"Evaluation of Islanding Detection Methods for Utility-Interactive Inverters in Photovoltaic Systems", SANDIA Report SAND2002-3591, Albaquerque, NM: Sandia Netional Labd, November 2002, available http://www.doc.gov/bridge 